\title{
Correlative 3D Imaging and Characterization of Human Dentine
}

Isabel N. Boona ${ }^{1}$, Frank Scheltens ${ }^{1}$, John Sosa $^{2}$, Timothy L. Burnett ${ }^{3}$, Phil J. Withers ${ }^{3}$, Jonathan S. Earl ${ }^{4}$, David W. McComb ${ }^{1}$

${ }^{1}$ Center for Electron Microscopy and Analysis, The Ohio State University, Columbus, OH, USA

${ }^{2}$ Center for the Accelerated Maturation of Materials, The Ohio State University, Columbus, OH, USA

${ }^{3}$ The University of Manchester, Materials Department, Manchester, UK

${ }^{4}$ GlaxoSmithKline Consumer Healthcare R\&D, Weybridge, Surrey, England, UK

3D characterization $[1,2]$ is of particular importance in the study of mineralized tissues such as teeth and bones due to the presence of channels, pores and features that span millimeter, micrometer and nanometer length scales. The major component in human teeth, by weight and volume, is dentine. This hydrated hard tissue encloses the central pulp and has microscopic channels, dentineal tubules that radiate from the pulp to the cementum on the surface of the dentine that connects with the hard outer enamel. The permeability provided by these tubules can cause dental hypersensitivity. The object of our current work is to understand how treatments for dental hypersensitivity act on these tubules.

This requires and understanding of chemical changes on the nanometer scale using techniques such as scanning transmission electron microscopy (STEM) combined with analytical methods such as energy dispersive spectroscopy (EDS) and electron energy loss spectroscopy (EELS). In particular, techniques such as X-ray computed tomography (micro-CT/nano-CT), S/TEM-HAADF imaging, Focused Ion Beam (FIB) sample preparation, Super-X XEDS and dual-EELS have been used to investigate the ultrastructure and chemistry of the human dentine specimen. Results on the 3D characterization of human dentine including the segmentation of dentine microtubules, peritubular dentine, as well as the role of dentine "nano-tubules" that branch off from the primary microtubules, will be discussed.

The human tooth sample was provided by GlaxoSmithKline Oral Healthcare, Weybridge, UK and was obtained with appropriate informed consent. Specifically the sample was a coronal dentine disc taken from below the enamel dentine junction and above the pulp cavity of the tooth by sectioning with a diamond tipped saw and the cut surfaces polished to a 2500 silicon carbide finish. The multi-scale correlative approach for this work consisted of five different steps at increasingly smaller length scales. The multi-scale correlative approach, seen in Figure 1, are divided into subcategories: a) Whole Dentine b) Micro-drilled (MD) Dentine c) Pillar d) Micro-cylinder and e) TEM sample. When the datasets were collected, organized, and prepped for correlation, the combination of MIPAR ${ }^{\mathrm{TM}}$ and Avizo ${ }^{\mathrm{TM}}$ software programs were used to combine all of the 3D visualization datasets. The volume - of -interest was located and visualized across all five datasets in video format.

In order to reliably and reproducibly identify the specific region and orientation for the FIB-SEM studies we utilized 3-D datasets using micro-CT and nano-CT methods from dentine specimens with suitable fiducial markers. Following XMT examination and FIB sample preparation, a monochromated Titan ${ }^{\mathrm{TM}}$ 60-300 STEM equipped with a Super-X XEDS collection system was used to investigate the dentine at the atomic scale.

This work has resulted in unparalleled characterization of human dentine at four different length scales. The combination of micro-CT, nano-CT, FIB, Super-X XEDS, has been applied successfully to characterize the same region of dentine from centimeters to the atomic scale. Ultimately, a 3-D correlative workflow will be used in clinical trials to evaluate both qualitatively and quantitatively the 
effectiveness of interventions on dental hypersensitivity from the atomic to the macroscopic scale [3].

References:

[1] Earl, J. S. et al, Journal of microscopy 240.1 (2010), p. 1.

[2] Parkinson, Charles R., and Alexander Sasov, Dental materials 24.6 (2008), p. 773.

[3] The authors acknowledge funding from Glaxo-Smith Klein. Dr. J. Earl is thanked for his many useful discussions and contributions to this work.

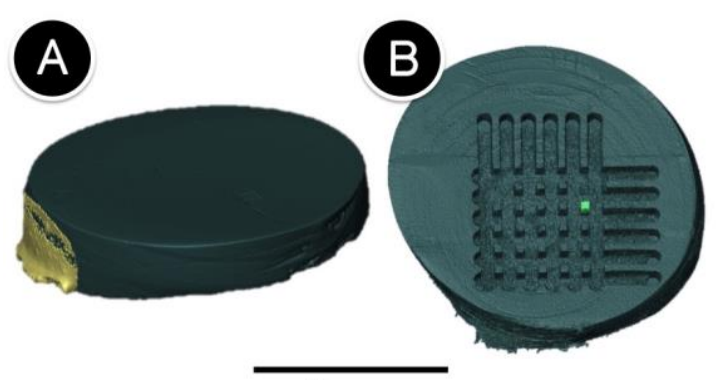

$5 \mathrm{~mm}$

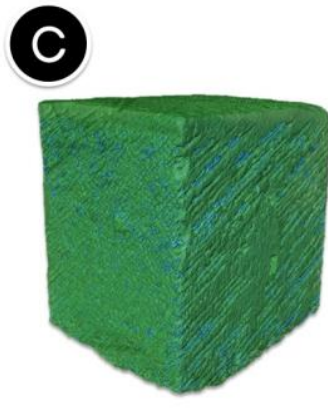

$100 \mu \mathrm{m}$

\section{D}

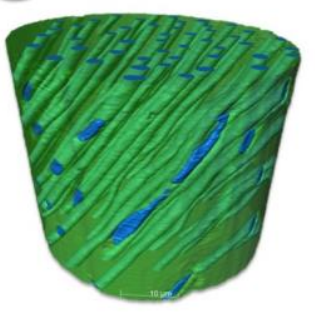

$\overline{10 \mu \mathrm{m}}$

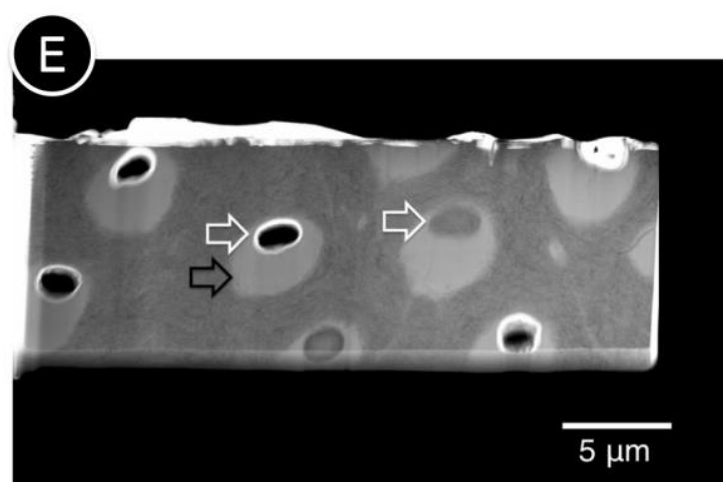

Figure 1. Multi-scale approach characterizing human dentine samples. (a) Whole Dentine; XMT (b) Micro-Drilled Dentine; XMT (c) Pillar; XMT (d) Micro-Cylinder; Nano-CT (e) Thinned TEM sample; FIB prep and HAADF STEM imaging, where the white arrows depict tubules and the black arrow depicts the peritubular dentine. 